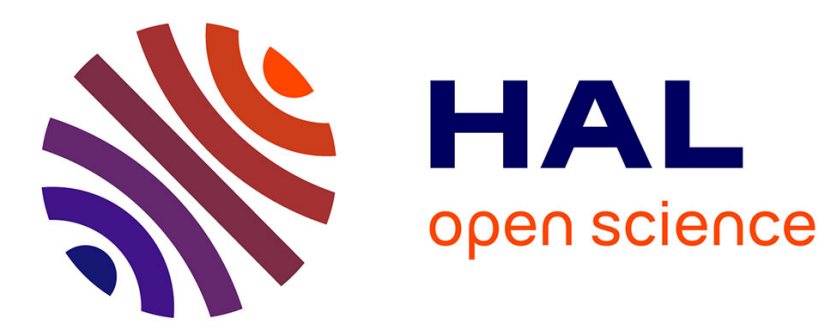

\title{
INDICES TEMPORELS SEGMENTAUX ET SOUS-SEGMENTAUX DANS LA PRODUCTION DES CONSONNES OCCLUSIVES DU FRANCAIS
}

\author{
S. Santi
}

\section{> To cite this version:}

S. Santi. INDICES TEMPORELS SEGMENTAUX ET SOUS-SEGMENTAUX DANS LA PRODUCTION DES CONSONNES OCCLUSIVES DU FRANCAIS. Journal de Physique IV Proceedings, 1992, 02 (C1), pp.C1-287-C1-290. 10.1051/jp4:1992161 . jpa-00251232

HAL Id: jpa-00251232

https://hal.science/jpa-00251232

Submitted on 1 Jan 1992

HAL is a multi-disciplinary open access archive for the deposit and dissemination of scientific research documents, whether they are published or not. The documents may come from teaching and research institutions in France or abroad, or from public or private research centers.
L'archive ouverte pluridisciplinaire $\mathbf{H A L}$, est destinée au dépôt et à la diffusion de documents scientifiques de niveau recherche, publiés ou non, émanant des établissements d'enseignement et de recherche français ou étrangers, des laboratoires publics ou privés. 


\title{
INDICES TEMPORELS SEGMENTAUX ET SOUS-SEGMENTAUX DANS LA PRODUCTION DES CONSONNES OCCLUSIVES DU FRANÇAIS
}

\author{
S. SANTI \\ Laboratoire "Parole et Langage", UA CNRS 261, 29 Av R. Schuman, F-13621 Aix-en-Provence, \\ France
}

ABSTRACT:

$t h$ is well known that segmental duration can be considered as a major acoustic cue. Specific durations of sounds are often explained as the output of articulatory and/or aero-dynamic constraints. In this paper, it is demonstrated that segmental duration plays a role in the acoustic discrimination of speech sounds for the voiced/unvoiced feature and place of articulation. The cues can be extracted directly from the duration of the segments and/or in acoustical events such as burst or occtusion phase. A statistical analysis supports our acoustical analysis. Aero-dynamic, phonofogical and percoptual explanations of the results are proposed.

\section{INTRODUCTION}

L'étude des variations temporelles de la chaine segmentale constitue, depuis longtemps, un champ dinvestigation privilégié (voir, par exemple, Di Cristo, 1978; O'Shaughnessy, 1981, Klatt 1976; Wajskoo, 1979; Raphaël 1981, Lisker, 1974; Durand 1985, etc). Aucun phénomène de producition ou de perception ne peut se concevoir en detors de cette dimension: la parole est construite of perçue dans le temps. Sous les termes de duré segmentale et sous-segmentale, vont se ranger divers phénomènes, contraints ou non-contraints physiologiquement, pertinents ou non-pertinents perceptivement, correspondant à un découpage (segmentation) de nos unités d’analyse de base, une série de logatomes de type VCV. Nous pouvons fournir, à priori, une décomposition temporelle de ces unités en différents niveaux (figure 1). La durée segmentale (of sous-segmentale) peut-elle ètre un indice acoustique pertinent dans la perception des consonnes occlusives? Si oui, de quelle manière? Quel sont les traits ainsi mis en opposition? Quels sont les segments ou sous-segments concernés?

Cefte recherche s'inscrit dans le cadre d'une étude portant sur l'extraction d'indices acoustiques, à partir de parole naturelle ef de leur application en synthese vocale. De ce lait, nos objectils sont multiples.

(a) Observation du comportement temporel des unités segmentales, analyse en termes de traits et d'indices, c'est à dire aspect acoustique et production.

(b) Implications au niveau de la percoption de la parole.

(c) Application éventuelle de régles issues de tanalyse, en synthèse vocale, modélisation des résultats de (a). Tests de perception (intelligỉilité, nalurel, etc), validation indirecte des hypothèses de (b) et de la méthode de modélisation (règles).

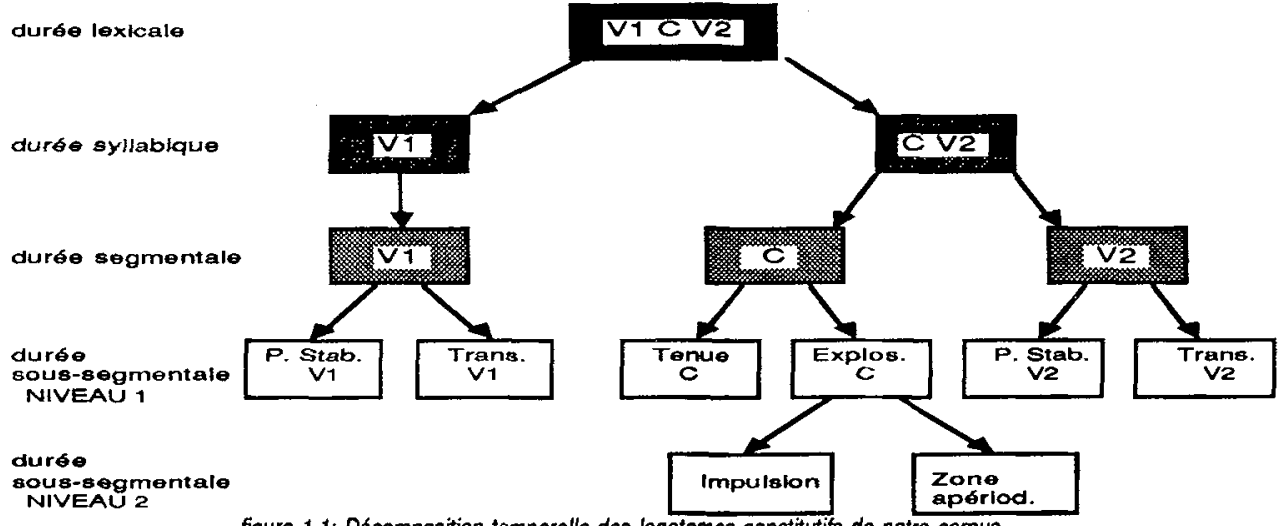

figure 1.1: Décomposition temporelle des logatomes constitutifs de notre corpus

\section{METHODOLOGIE}

Notre copus se compose de logatomes de type VCV, de structure vocalique symétrique où $V$ est une des voyelles cardinales /a, $i$ $u /$ et $C$ une des consonnes occlusives du français $t, d, g, p, t, k$, soit au total 18 logatomes. Ce corpus à été enregistré en chambre anéchoíque par deux locuteurs. Les logatomes ont été produits de façon isolée (sans phrase porteuse). 
Bien que nous ne présentions pas ici les règles issues de cette étude, il est utile de savoir que le synthátiseur utilisé est d'un type permettant cette implantation de manière trés souple. Notre synthétiseur est de type paramétrique à formants, semblable à celui proposé et developpé par Klatt (1980).

La segmentation temporelle à été conduite à l'aide d'un logiciel de trailement du signal de parole, implanté sur un mini-ordinatteur MASSCOMP 5400, réalisé par Robert Espesser, au Laboratoire Parole et Langage à Aix en Provence. Cet outil autorise toutes les méthodes classiques utilisées pour la segmentation du signal de parole: tracés oscillographiques, sonagrammes, etc.

Notre analyse statistique (T-test de Student, analyse de variance et corrélations) ont été réalisées sur un micro-ordinateur Macintosh (connecté au mini-ordinateur).

La notion de mesure temporelle se superpose, dans notre perspective, à celle de segmentation. Mesurer un segment ou un soussegment, c'est déjà faire une hypothèse sur la nature acoustique intrinséque du segment considéré (y compris dans ses autres dimensions). La recherche d'indices de segmentation peut être considérée comme une analyse qualitative préalable du signal. Le cas qui nous préoccupe n'est pas, en théorie, et selon les spécialistes en segmentation, le plus difficile à traiter (contrairement à la détermination des frontières entre des segments présentant des modes d'articulation proches ou identiques, par exemple Ayil, /mwal, etc ...). II est à noter que, pour notre analyse des durées, l'homogénéité dans le choix d'un critère est tout aussi important que le critère en lui-même.

\section{3- RESULTATS ET ANALYSE} à Santi (1992).

Les résultats de nos mesures et de notre analyse statistique sont présenté ici de manière sommaire, pour plus de détails, se reporter

Les durées globales (ou lexicales, cf figure 1) de nos lagatomes présentent une certaine homogénéité intra et inter-focuteur. Aucun stimulus ou groupe homogène de stimuli ne se délache de ces ensembles. Les durées moyennes sont en accord avec ce à quoi l'on peut s'attendre pour le type de parole mise en ceuvre (moyenne de $427 \mathrm{~ms}$, écart type de $27 \mathrm{~ms}$ ). Un t-Test, pour l'opposition voisé/non-voisé, et une analyse de variance, pour le lieu d'articulation, n'ont montré aucune opposition statistiquement significative sur les durées globales de nos logatomes.

\section{3-1. Oppositions temporelles segmentales et sous-segmentales sur les traits de volsement et de lieu d'articulatlon} 3-1-1- Trait de voisement

Oppositions segmentales

Une première opposition apparait: la durée vocalique de V1 semble quasi-systématiquement plus brève en contexte de consonne occlusive non-voisée que voisée. Celte opposition est significative. Cette même opposition est reprise à son compte par la voyelle V2, avec cependant un écart moyen et un seuil de significativité moindres. Bien entendu, l'ensemble de l'environnement vocalique (c'est à dire si ton additionne V1 à V2) manifeste une opposition signilicative entre les sous-classes voisé et non-voisé (tableau 1).

Les durées totales des deux sous-groupes voisé et non-voisé s'opposent également de façon significative au niveau de la durée du segment consonantique. De laçon inversée par rapport aux voyelles, c'est cette fois le sous-groupe non-voisé qui présente une durée supérieure. Cette opposition est constante pour l'ensemble des stimuli (tableau 1).

\section{Oppositions sous-segmentales}

L'examen, en parallèle, de la durée des tenues des segments consonantiques voisés et non-voisés ftoutes choses égales par ailleurs, locuteur, lieu d'articulation et environnement vocalique) confirme que nous avons une opposition systématique entre ces deux sousclasses. Les consonnes voisées ont donc une tenue significalivement plus bréve que les consonnes non-voisées (tableau 1).

Une opposition significative, au niveau de la durée de lexplosion, apparait entre les sous-ciasses voísé et non-voisá. La durée de l'explosion des stimuli non-voisés est toujours supérieure à celle des stimuli voisés, sauf pour les cas où les durées sont nulles, c'est à dire lorsque aucune explosion n'a été relevée lors de l'analyse. Ce phénomène montre que la durée participe à la différenciation des deux sous-classes dans la réalisation globale de la consonne (tableau 1).

\section{3-1-2- Lieu d'articulation}

Oppositions segmentales

La durée des segments vocaliques, que ce soit V1 ou V2, ne participe pas de façon significative à la discrimination (diun point de vue acoustique) entre les lieux d'articulation labial, dental et vélaire. Rappelons qu'en ce qui concerne les indices temporels relatís au trait de voisement, l'environnement vacalique se comportait de manière cohérente, c'est à dire en évoluant de façon opposée à la durée consonantique en général et à la durée des sous-segments "tenue" et "explosion". Nous ne sommes certes pas ici en présence d'un phénomène aussi caractéristique, c'est à dire régulier sur l'ensemble de l'environnement vocalique V1 et V2 et statistiquement significatil. Nous pouvons tout de même noter une cortaine "régularité" au niveau de V2, ou les durées respectives des lieux d'articulation se rangent de la façon suivante: LABIAL $<$ DENTAL < VELAIRE.

tableau 1: t-Tests de Student sur lopposition voisé/non-voisé, au niveau de la durée des segments V1, V2 et $C$, des soussegments Tenue ef Explosion, et de la combinaison V1+V2.

\begin{tabular}{|c|c|c|c|c|}
\hline & $\begin{array}{l}\text { Gcart } \\
\text { moyen }\end{array}$ & valour $t$ & probabilits & $\begin{array}{l}\text { dogrés } \\
\text { liberte }\end{array}$ \\
\hline$\vee 1(v / n v)$ & 24.83 & 7.7 & 1.0OOOE-4 & 17 \\
\hline Ve (V/nv) & 14.11 & 2.98 & .0084 & 17 \\
\hline$V_{1}+V_{2}(v / n v)$ & $\mathbf{3 8 . 9 4}$ & 5.86 & 1.OOOOE-4 & 17 \\
\hline$O(v / n v)$ & -35.78 & -7.65 & 1.000OE-4 & 17 \\
\hline Tenue (vinv) & -15.28 & -3.54 & 0.0025 & 17 \\
\hline Expl. (v/riv) & -20.5 & -5.47 & 0.0001 & 17 \\
\hline
\end{tabular}

Une analyse de variance a été réalisée pour la durée segmentale de la consonne en fonction du lieu ơarticulation (L1 et L2 traités conjointement). Si une variabilité impontante (et statistiquement signifficative,) est présente entre les stimuli (de façon globale, c'est-à-dire sans tenir comple de la variable "lieu d'articulation"), aucune opposition sur le lieu d'articulation n'apparaít. 
Oppositions sous-segmentales

Les résultats montrent une difiérence systématique entre les moyennes des durées des tenues pour chaque lieu d'articulation, ceci quel que soit l'environnement vocalique. L'analyse de variance montre une différence significative entre les durées des tenues en fonction du lieu d'articulation (tableau 3). L'examen des comparaisons montre que seule l'opposition entre les lieux labial ef vélaire est statistíquement significative (il s'agit des valeurs extrêmes). Cependant, la hiérarchie suivante, construite à partir des moyennes, peut étre retenue: LABIAL > DENTAL > VELAIRE

Les durées d'explosion participent à la discrimination globale du lieu d'articulation. L'examen de l'analyse de variance montre que la variable "lieu d'articulation" présente un effet global significatif (tableau 4). Cependant, seules les oppositions labial/dental et labial/vélaire sont responsables de cel effet. Nolons touteíois que si ropposition dental/vélaire n'est pas statistiquement signilicative, ceite derniere participe de façon cohérenle à l'établissement de la hiérarchie suivante: LABIAL < DENTAL < VELAIRE. Parmi les stimuli s'opposant sur le lieu d'articulation (au niveau de la durée de lexplosion), c'est principalement les éléments non-voisés qui sont responsables des variations les plus importantes.

\section{3-2- Corrélations entre durées vocaliques et durée consonantique}

Notre hypothèse, à la lumière des résultals observés (en ce qui concerne le paramètre de durée en tant qu'indice, aussi bien du trait de voisement, que du lieu d'articulation), est que, globalement, la durée consonantique s'organise de manière inversée par rapport à la durée vocalique. Nous avons examiné la validité de cette affirmation à l'aide d'une analyse des corrélations. Pour chaque corrélation envisagée, un élément de type consonantique (durée segmentale ou durée de la tenue) à été associé à un élément de type vocalique (V1, V2 ou V1 $+V_{2}$ ) (tableau 2).

\section{DISCUSSION}

Le faux problème des sevils différentiels de durée

Nous avons tenu, lors de la présentalion des résultats de notre analyse temporelle, à ne pas faire intervenir de critères de pertinence issus d'une interprétation perceptuelle. II semble cepenciant évident qu'un phénomène, mème statistiquement significatif, ne trouve son utilité en synthèse vocale, que dans la mesure où ce dernier est susceptible d'être perçu. Le critère le plus utilisé pour démontrer la validité perceptive des modifications de durée est le seuil diftérentiel de durée. Cependant, nous avons choisi de ne pas directement tenir compte de ce critère. En effet, ces expériences nous renseignent sur la capacité des sujets à rendre compte d'un phénomène perceptif conscient, mais ne peuvent prétendre, en aucun cas, décrire lutilisation du paramètre de durée pendant le(s) processus mis en oeuvre lors du décodage acousticophonétique. Rien ne nous autorise à prótendre qu'un seuil déterminé lors d'une tâche donnée pourra s'appliquer lors d’une tâche différente. En d’autres termes, les sujels peuvent très bien utiliser des dilférences de durées inférieures aux seuils el ne pas avoir conscience de ce phénomène.

tableau 2: Résultats de l'analyse des corrélations entre diverses combinaisons de segments et sous segments (colonne de gauche)

\begin{tabular}{|c|c|c|c|c|}
\hline & $\boldsymbol{F}$ & $\mathbf{R}^{2}$ & probabilits & $\begin{array}{l}\text { type } \\
\text { correl. }\end{array}$ \\
\hline$V_{1}+V_{2} / C$ & 537 & 288 & $P<.0007$ & nogative \\
\hline$\vee 1 / C$ & .549 & 301 & $P<.0005$ & negative \\
\hline$V_{2} / C$ & 374 & 14 & $p<.025$ & negative \\
\hline$V_{1}+V_{2} /$ tenue & .35 & .123 & $p<.04$ & negative \\
\hline$V 1 /$ tenue & 376 & .141 & $p<.024$ & nógativo \\
\hline$v_{2} /$ tenue & .26 & .067 & $n s$ & nógative \\
\hline
\end{tabular}

Une interprétation perceptive des relations temporelles voyelle/consonne

De nombreuses théories, supposées rendre comple des rapports temporels entre consonnes et voyelles décrits ci-dessus, ont été élaborées sans qu'aucune ne fasse l'unanimité. La plupart de ces dernières sont de type articulo-phonatoire (pour un compte rendu critique détaillé, voir Di Cristo 1985). Chen (1970) envisage également, à la suite d'idées formulées par Denes (1955) et Lisker (1957), une interprétation de type perceptit. Les modifications de la durée vocalique en fonction du caractère voiséinon-voisé de la consonne adjacente seraient destinées à optimiser la discrimination de la durée de l'occlusion des deux modes phonatoires respectifs. Cette idée a été reprise et développée par Kluender et al. (1988). Pour ces auteurs, le système linguistique génère une durée vocalique systématiquement inverse par rapport à la durée de locclusion afin de renforcer perceptivement cette dernière. Nous pensons que cette hypothèse est particulièrement intéressante. En eftet, il est a été montré, notamment pour la vision, que la perception d'un phénomène continu est dépendante du contexte d'apparition de ce phénomène: un même ton de gris est perçu plus foncé sur un fond blane et plus clair sur un fond noir. Ce principe de mise en relief par le contraste a également été démontré pour la perception de la hauteur tonale. Dans le cas qui nous intéresse, nous devons prendre en compte l'ińluence mutuelle de chacune des composantes. La durée de la voyelle inllue sur la perception de la durée de la tenue et réciproquement, chacune étant simultanément figure et fond (figure 2).

La durée de la tenue, éléments aérodynamiques, articulatoires et phonologiques La contrainte imposée par le maintien en vibration des cordes vocales, pendant la tenue de l'occlusion des consonnes acelusives sonores, concourt certainement à produire une tenue plus brève que lorsque cette contrainte est absente, ce qui est le cas des consonnes occlusives sourdes. Par contre, nous n'avons, jusqu'ici, fourni aucune hypothèse à propos des différences de durée impulables au lieu d'articulation.

Nous pensons qu'il est possible que le raccourcissement de la durée de la tenue de locelusion des consonnes voisées soit également imputable aux contraintes aérodynamiques régissant la production de ce type de consonnes. Il est établi que la brièvelé de la tenue de l'occlusion du /g/est due à la position reculée de son lieu d'articulation. Les pressions sous-glottique et sub-glottique s'équilibrant plus rapidement, à cause de la taille plus réduite, à cet instant, du conduit vocal, la vibration des cordes vocales ne peut se prolonger. On assiste donc, soit à un dévoisement, soit à un "abrègement". 


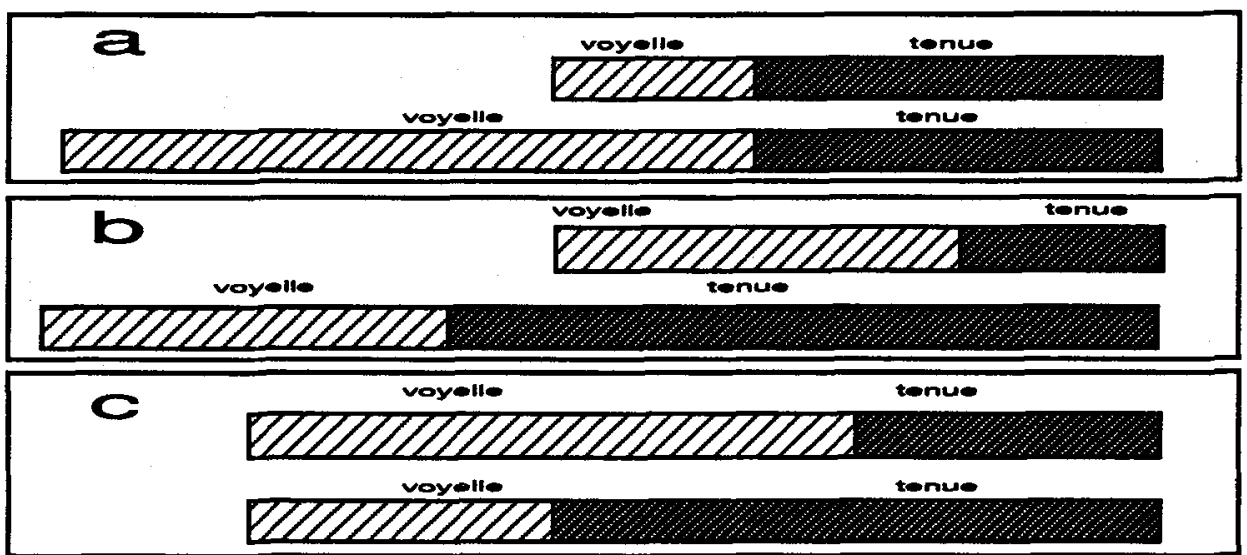

figure 2: a) Une voyelle brève ou longue va avoir tendance à modifier la perception d'une tenue de durée identique; b) Le méme phenomène se produit pour une voyelle de durée identique accompagnée dune tenue bréve ou longue; c) Les deux effets potentiels sont cumulés, ropposition contrastive est maximale. C'est ce demier cas de figure (c) qui schómatise lo mieux lo résultat de notre analyse.

Une des particularités d'un corpus de logatomes, est le soin avec lequel lo ou les locuteurs réalisent la láche demandée (en principe...). Cette attention particuliàre à "bien prononcer" peut très bien bloquer la solution du dévoisement, le locuteur sachant, de façon intuitive, que ce phénomène est "stranger" à la forme canonique du /g/; la solution de rabrégement est alors la seule possible. En parole spontanée ou en lecture à débit rapide, celte hypothèse serait difficilement défendable. La production du phonème /d/est également régie par les mèmes contraintes articulo-aérodynamiques, à la seule différence que, le point d'occlusion étant plus avancé, lo conduit vocal présente un volume plus important que pour $/ g /$, léquilibre des pression se fait plus tard, donc le locuteur est susceptible de produire une tenue plus longue. Ce principe s'applique également entre $/ d /$ et $/ \mathrm{b} /$.

Si celte explication est valable, dans une situation de production particulière, pour le sous-ensemble "voise" des consonnes occlusives du français, elle ne peut en aucun cas, pour les consonnes non-voiseos, expliquer directement la réduction de la durée de la tenue en fonction du recul du point darticulation. La seule explication possible pour expliquer ce phénomène, serait alors le comportement du système phonologique. Le comportement lemporel de la sous-classe "non-voise" serait calqué, en fonction du lieu darticulation, sur celui de la sous-classe "voisé". Il est plus économique pour le système de gérer les indices de laçon cohérente, quitte à s'appuyer sur des indices non-contraints, c'est à dire plus "artificiels".

\section{5- CONCLUSION}

Il est aujourd'hui altesté que la durée des segments (phonèmes) of sous-segments (par exemple la tenue ou l'explosion dune consonne occlusive) constitutifs de la parole, possède un stalut dindice acoustique majeur. La durée spócifique de classes de sons (ou de sousclasses) est le plus souvent considérée comme issue de contraintes phono-articulatoires et/ou aéro-dynamiques. A partir d’une série de mesures temporelles segmentales et sous-segmentales, portant sur un corpus de logatomes de type VCV (où $V$ est une des voyelles cardinales $/ a /, N, i u /$ et $C$ une des consonnes occlusives du français $/ \mathrm{pl}, \mathrm{N}, \mathrm{NJ}, \mathrm{M} /, \mathrm{d} /, \mathrm{g} /$ ) nous avons montré que la durée joue un rôle d'indice au niveau de la discrimination entre les sous-classes consonnes occlusives voisées el non-voisées ainsi qu'entre les lieux d’articulation considérés: labial, dental et vélaire. L'indice temporel de discrimination du trait de voisement se retrouve tant au niveau des durées des segments vocaliques que des sous-segments "lenue de locclusion" ef "explosion". Une interprétation des résultals observés a été proposée, cette dernière taisant appel, à la fois aux argumentations traditionnelles (au niveau contraintes de production), mais également à une approche plus originale faisant intervenir les phénomènes de contraste subjectif inhérents aux processus généraux de la perception.

\section{REFERENCES}

Chen, M., 1970. "Vowel length variation as a fundion of the consonant environment", Phonetica 22, 129-159.

Denes, P., 1955. "Effect of duration on the perception of voicing", J.A.S.A. 27, 761-764.

DI Cristo, A., 1985. "De la microprosodie à lintonosyntaxe", Editions du CNRS, Publications de l'Universilé de Provence, Alx en Provence.

Durand, P., 1985. "Variabilíé acoustique et invariance en français, consonnes occlusives ot voyelles, Editions du CNRS, Publications de PUniversité de Provence, Aix en Provence.

Klatt, D., 1976. "The linguistic uses of segmental duration in english: acoustic and perceptual evidence", JA.S.A. 44/2, $401-407$.

Klatt, D., 1980. "Software for a cascade/parallel synthesizer", J.A.S.A., 67/3, 971-995.

Kluender, K., Diehl, R., Wright, B., 1988. "Vowel-lenght differences belore voiced and voiceless consonants: an auditory explanation", J. of Phonet., 16/2, 153-171.

Lisker, L., 1957. "Closure duration and the intervocalic voicad/unvoiced distinction in english", Language 33, $42-49$.

Llsker, L., 1974. "On explaining vowel duration variation", Slatus Report on Speech Research 23, 225-232.

O'Shaughnessy, D., 1981. "A study of trench vowel and consonani durations", J. of Phonet. 9.

Raphaeil, L., 1981. "Durations and contexts as cues to word final cognate opposition in english, Phonetica 38, 126-127.

Santi, S., 1992. "Synthèse vocale du français: modélisation acoustique ot évalualion perceptive", Thèse de Doctoral, Aix en Provence.

Wajskop, M., 1979. "Segmental durations of french intervocalic plosives", in Frontiers of Speech Communication, Lindblom \& Ohman Eds, Acadómic Press.

Les tableaux 3 ot 4 ne pouvent flgurer, faute de place, ils sont disponibles sur simple demande 